\title{
A Holistic Intervention for Oral Lichen Planus
}

\author{
Prashanth Panta ${ }^{1}$, Archana Andhavarapu ${ }^{2}$, Shankargouda Patil ${ }^{3}$
}

The Journal of Contemporary Dental Practice (2019): 10.5005/jp-journals-10024-2635

Oral lichen planus (OLP) is a chronic immune-mediated, inflammatory, and psychosomatic condition that frequently affects the oral mucosa in a typical bilateral pattern, often noticed in middle-aged females. ${ }^{1,2}$ It involves the buccal, lingual, and/or gingival mucosa and is also an oral potentially malignant disorder (OPMD). ${ }^{2}$ OLP is associated with a range of etiological factors from viruses like hepatitis $C$, to preventable local factors like dental plaque/calculus, to deep rooted, often underestimated, psychological triggers. ${ }^{1}$ OLP is routinely encountered in oral medicine clinics and is sometimes accompanied by the simultaneous involvement of skin, nails, genitals, and/or mucous membranes. Although OLP is multifactorial in origin, a large share of these presentations can be attributed chiefly to psychological factors like stress, anxiety, and depression. OLP is even more striking as compared to other conditions as its genesis, exacerbation, and progression closely mirror the dynamics of emotional disturbance.

As many OLP patients frequently display psychological distress, anxiety, depression, and disturbed sleep pattern, ${ }^{3-5}$ it is highly recommended that clinicians fully capture these contributory factors through relevant assessment instruments/questionnaires. It is certainly important to screen for these root-level triggers, and a detailed and meticulous history can retrieve this vital information. This will further enable clinicians to holistically evaluate and treat patients based on the predominant emotional factor, and not solely through pharmacological interventions! A survey also documented the positive benefit of social support and spirituality as protective for OLP patients. ${ }^{6}$ Although topical corticosteroids are the gold-standard therapy for OLP, additional emotional training will maximize the benefit to these patients

In a recent systematic review on salivary biomarkers in OLP, $55.5 \%$ of selected studies showed higher salivary cortisol (stress marker) levels in OLP group over healthy controls, ${ }^{7}$ and some studies have also revealed a linear relationship between severity of clinical disease and serum cortisol elevation. ${ }^{8}$ Consistent elevation in cortisol is clearly elucidating the psychosomatic origins of OLP. This scenario is pointing at the potential of mindbody interventions like meditation toward holistic rehabilitation. Despite the widely reported benefits of meditation, no research group pursued it in the context of OLP, an important gap in the therapeutic literature. Therefore, in this editorial, we have provided a theoretical framework of meditation and its beneficial effect on different aspects of "OLP," an established psychosomatic entity.

Meditation works through "awareness" ${ }^{\text {" }}$ and inculcates in its practitioner the attitude of "living-in-the-present," which is highly relevant to patients suffering from psychosomatic disorders. Meditations cause a paradigm shift in thinking/thought process and can impede the inception, generation, and perpetuation of deranged mental states like stress, anxiety, and depression.
${ }^{1}$ Department of Oral Medicine and Radiology, MNR Dental College and Hospital, Sangareddy, Telangana, India

${ }^{2}$ Department of Pulmonary Medicine, Malla Reddy Medical College for Women, Hyderabad, Telangana, India

${ }^{3}$ Department of Maxillofacial Surgery and Diagnostic Sciences, Division of Oral Pathology, College of Dentistry, Jazan University, Jazan, Kingdom of Saudi Arabia

Corresponding Author: Prashanth Panta, Department of Oral Medicine and Radiology, MNR Dental College and Hospital, Sangareddy, Telangana, India, Phone: +91 9701806830, e-mail: maithreya.prashanth@gmail.com

How to cite this article: Panta P, Andhavarapu A, et al. A Holistic Intervention for Oral Lichen Planus. J Contemp Dent Pract 2019;20(7): 765-767.

Source of support: Nil

Conflict of interest: None

Meditation practice enables individuals to devote their full mind to the present moment and minimizes disturbance from "thoughtsof-the-past" and "imaginations-of-the-future." ${ }^{\prime 9,10}$ The phenomenon of "suspending awareness" (in present moment) is referred as "mindfulness," which is the essence/heart of all meditations. ${ }^{9}$ The neurophysiological correlates of all meditation methods is similar and can be attributed primarily to "awareness/mindfulness," their working principle. Furthermore, meditation is easy-to-practice and is a nonpharmacological solution to chronic psychosomatic diseases that have deeper psychological and emotional roots. It is, therefore, logical to pursue such rooted yogic interventions, at least as an adjuvant strategy for OLP., 911,12

Anxiety is "unrealistic worry" and is a common observation in OLP patients. In fact, the severity-of-symptoms correlated with anxious and depressive symptoms even in symptomatic-reticular OLP cases. ${ }^{13}$ On the contrary, many clinicians may be considering only erosive-OLP to be linked to psychological distress, which is not the case. Anxiety levels critically decline following meditation due to enhanced activity in ventrolateral and ventromedial portions of prefrontal cortex, and due to increased connectivity between prefrontal cortex (PFC) regions and amygdala. ${ }^{11}$ Long-standing meditation practice induces "emotion regulation," a key event that underlies the wide-spread benefits of meditation. Emotional regulation can improve the quality-of-life (QoL) because of overall reduction in impulsivity and also improves the ability to quickly reach to baseline. The neural processes that underlie emotional regulation include diminished activation of amygdala. Irrespective of the subtype of OLP, anxiety and depression should be considered as root-level triggers, and meditation methods can be formulated as a useful complementary strategy. ${ }^{9,11,12}$ 
Poor "quality-of-sleep" is another frequent observation in OLP patients. Sleep disturbances in OLP can be primarily due to underlying stress, anxiety, and depression ${ }^{3,4}$ that can result from mental, emotional, and/or financial crisis. ${ }^{5}$ Meditation influences important sleep parameters like slow-wave and rapid-eyemovement (REM) sleep. Interestingly, studies have shown that older meditators can retain a sleep pattern similar to younger adults! ${ }^{14}$ In fact, mindfulness meditation was suggested as an auxiliary treatment for sleep disorders. ${ }^{15}$ In accordance with several references, meditation can undoubtedly improve quality-ofsleep, amount-of-sleep time, and can also reduce need-for-sleep (Flowchart 1)..$^{9,15-17}$

Furthermore, meditation may alter the dynamics of OLP disease through modulation of "inflammation" and "immunity." In the recent times, there is expanding evidence on the dose-dependent effect of meditation on different immune system parameters like immune cell count, immune cell aging, antibody response, etc. ${ }^{19}$ Meditation can cause downregulation of nuclear factor kappa beta (NF-k $\beta$ ) pathway ${ }^{18}$ and can also reduce circulating C-reactive protein (CRP) levels, which are linked to "cancer-related inflammation" and "systemic inflammation," respectively. Meditation also elevates $\mathrm{CD}^{+}{ }^{+} \mathrm{T}$ cell count/activity and increases telomerase activity. ${ }^{19}$ During the recent times, "telomere shortening" was consistently observed in biological aging, cancer, and several chronic diseases. Surprisingly, meditation has demonstrated a "telomere-lengthening effect" in numerous studies, which is a marker of cellular well-being. ${ }^{19}$ Due to its overall effect on NF-k $\beta$

Flowchart 1: Role of psychological triggers in OLP and possible beneficial effect through meditation

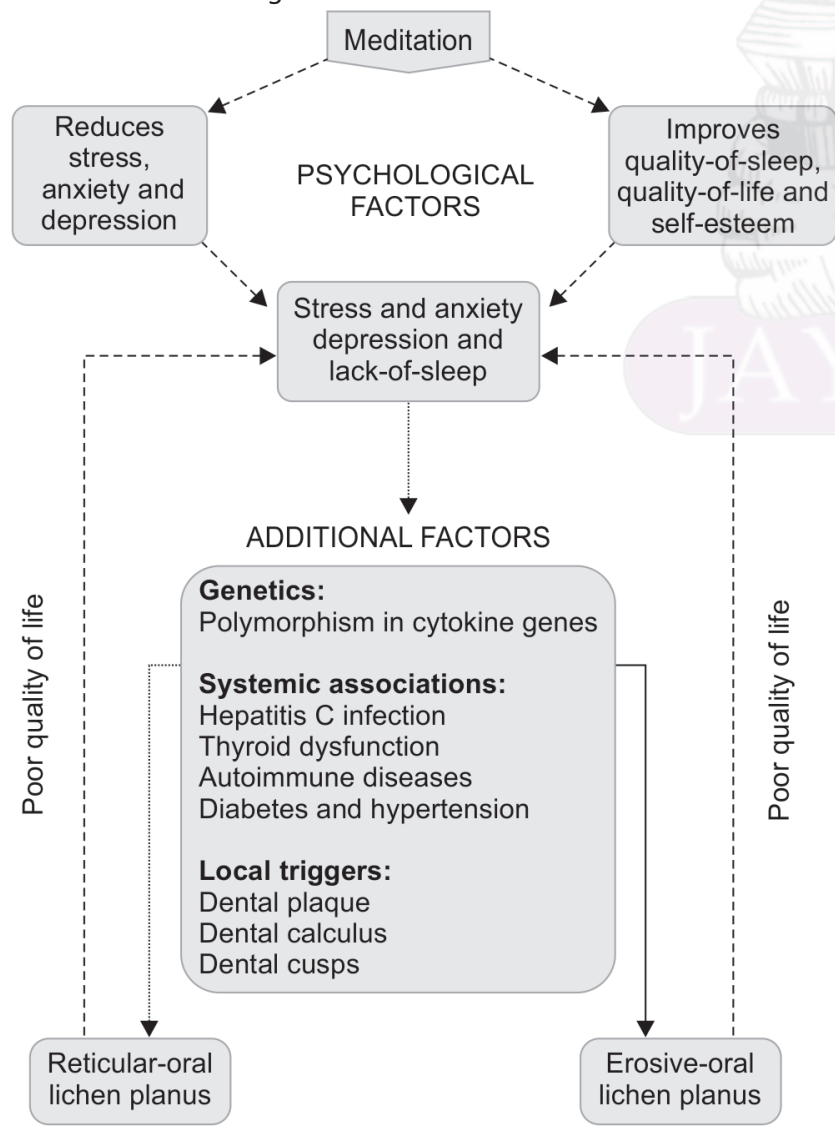

activity and other inflammatory pathways, immunity, and telomere length, meditation can benefit OLP patients at the tissue, cellular, and subcellular scales. ${ }^{20}$

As meditation positively influences a wide-spectrum of psychological triggers/factors that are often reported in OLP, $, 11,12$ it would cause holistic rehabilitation, an aspect that was not addressed by previous therapies. "Mindfulness," the working principle of meditation, enables individuals to be more wakeful/ aware, and conscious of the present moment, offering ultimate protection from psychological and emotional distress. ${ }^{1}$ In the present moment, deranged mental states like stress, anxiety, and depression are obliterated, causing spontaneous rehabilitation at the root level. Meditation is, therefore, a supreme method of neuro-modulation that could enable mental and emotional balance, and spiritual wellness and tranquility among OLP patients, thus, arresting existing symptoms and also thwarting occasional exacerbations. Meditation significantly reduces stress, anxiety, and depression; improves quality-of-sleep; reduces blood cortisol levels; ${ }^{12}$ modulates inflammation and immunity; and remarkably improves the QOL through awareness and equanimity. ${ }^{9,11,12}$

As OLP is orchestrated and compounded by stress, anxiety, depression, and sleep problems, we firmly believe that mind-body interventions like meditation can be a promising strategy to prevent and/or reverse clinical disease and to rehabilitate suffering patients effectively. Apart from OLP, oral psychosomatic conditions like burning mouth syndrome (BMS), ${ }^{21}$ temporomandibular disorders including myofascial pain dysfunction syndrome ${ }^{9}$ may be greatly benefited through mind-body interventions.

\section{References}

1. Eisen D. The clinical features, malignant potential, and systemic associations of oral lichen planus: a study of 723 patients. J Am Acad Dermatol 2002;46:207-214. DOI: 10.1067/mjd.2002.120452.

2. Panta $P$, Andhavarapu A, et al. Reverse Koebnerization in a linear oral lichenoid lesion: A case report. Clin Pract 2019;9:1144. DOI: 10.4081/ cp.2019.1144.

3. Vallejo MJ, Huerta G, et al. Anxiety and depression as risk factors for oral lichen planus. Dermatology 2001;203:303-307. DOI: 10.1159/000051777.

4. Chaudhary S. Psychosocial stressors in oral lichen planus. Aust Dent J 2004;49:192-195. DOI: 10.1111/j.1834-7819.2004.tb00072.x.

5. Adamo D, Ruoppo E, et al. Sleep disturbances, anxiety and depression in patients with oral lichen planus: a case control study. J Eur Acad Dermatol Venereol 2015;29:291-297. DOI: 10.1111/jdv.12525.

6. Rana M, Kanatas A, et al. Relevance of psychosocial factors to quality of life in oral cancer and oral lichen planus: a prospective comparative study. Br J Oral Maxillofac Surg 2015;53:621-626. DOI: 10.1016/ j.bjoms.2015.04.007.

7. Humberto JSM, Pavanin JV. Cytokines, cortisol, and nitric oxide as salivary biomarkers in oral lichen planus: a systematic review. Braz Oral Res 2018;32:e82. DOI: 10.1590/1807-3107bor-2018.vol32. 0082.

8. Shah B, Ashok L, et al. Evaluation of salivary cortisol and psychological factors in patients with oral lichen planus. Indian J Dent Res 2009;20:288-292. DOI: 10.4103/0970-9290.57361.

9. Panta P. The Possible Role of Meditation in Myofascial Pain Syndrome: A New Hypothesis. Indian J Palliat Care 2017;23:180-187. DOI: 10.4103/0973-1075.204239.

10. Panta P. 'Meditation Training Intervention' - A necessary shift for head and neck cancer patients. Oral Oncol 2018;86:316-317. DOI: 10.1016/ j.oraloncology.2018.09.002.

11. Tang YY, Hölzel BK, et al. The neuroscience of mindfulness meditation. Nat Rev Neurosci 2015;16:213-225. DOI: 10.1038/nrn3916. 
12. Pascoe MC, Thompson DR, et al. Mindfulness mediates the physiological markers of stress: Systematic review and meta-analysis. J Psychiatr Res 2017;95:156-178. DOI: 10.1016/j.jpsychires.2017.08.004.

13. Adamo D, Cascone M, et al. Psychological profiles in patients with symptomatic reticular forms of oral lichen planus: A prospective cohort study. J Oral Pathol Med 2017;46:810-816. DOI: 10.1111/ jop.12577.

14. Nagendra RP, Maruthai N, et al. Meditation and its regulatory role on sleep. Front Neurol 2012;3:54. DOI: 10.3389/fneur.2012.00054.

15. Gong $\mathrm{H}, \mathrm{Ni} \mathrm{CX}$, et al. Mindfulness meditation for insomnia: A metaanalysis of randomized controlled trials. J Psychosom Res 2016;89:1-6. DOI: 10.1016/j.jpsychores.2016.07.016.

16. Silber MH. Clinical practice. Chronic insomnia. N Engl J Med 2005;353:803-810. DOI: 10.1056/NEJMcp043762.

17. Rusch $\mathrm{HL}$, Rosario $M$, et al. The effect of mindfulness meditation on sleep quality: a systematic review and meta-analysis of randomized controlled trials. Ann N Y Acad Sci 2019;1445:5-16. DOI: 10.1111/ nyas.13996.

18. Buric I, Farias $M$, et al. What is the molecular signature of mindbody interventions? A systematic review of gene expression changes induced by meditation and related practices. Front Immunol 2017;8:670. DOI: 10.3389/fimmu.2017 00670.

19. Black DS, Slavich GM. Mindfulness meditation and the immune system: a systematic review of randomized controlled trials. Ann N Y Acad Sci 2016;1373:13-24. DOI: 10.1111/nyas.12998.

20. Kiecolt-Glaser JK, Christian L. Stress, inflammation, and yoga practice. Psychosom Med 2010;72:113-121. DOI: 10.1097/ PSY.0b013e3181cb9377.

21. Feller L, Fourie J, et al. Burning Mouth Syndrome: Aetiopathogenesis and Principles of Management. Pain Res Manag 2017;2017:1926269. DOI: $10.1155 / 2017 / 1926269$. 